IP Periodica Polytechnica

Mechanical Engineering

57(1), pp. 13 19. 2013

DOI: 10.3311/PPme.7012

Creative Commons Attribution (i)

RESEARCH ARTICLE

\section{Influence of grid-to-rod fit on fuel rod fretting}

\author{
Szilárd Kovács, Jürgen Stabel, Mingmin Ren, Bruno Ladouceur
}

Received 2013-04-30

\begin{abstract}
The fretting behavior of nuclear fuel assemblies is influenced by a number of parameters. One of these is the fit between the fuel rod and the spacer grid, which changes during the fuel residence time in the core. The influence of fit on fretting has been investigated by performing model tests on a typical fuel rod support configuration. The test rod was suspended in water during the tests and was excited electromagnetically to induce vibration. Fretting tests each lasting 100 hours were carried out at room temperature for each individual condition of fit. The results showed that the first small fretting marks, having a maximum depth of $13 \mu \mathrm{m}$, did not start to appear until the spacer spring preload has been reduced to approximately 0.5-3.5 N. Substantial amount of wear was only observed in case of gapped support. The depth and volume of the fretting marks increased significantly as the preload decreased and the grid-to-rod gap increased. It was found that the stiffness of the vibrating system decreases as the size of this gap increases, allowing the test rod to move more freely and more extensively at the same level of excitation. It was further shown that the probability of fretting damage is greater with a clearance fit than with an interference fit (spring preload), and increases as the gap becomes larger. The results of this work are to be applied in the development of future spacer grids and should provide a better understanding of fretting processes inside the reactor.
\end{abstract}

\section{Keywords}

fretting wear $\cdot$ fretting depth $\cdot$ fretting volume $\cdot$ support condition

\section{Szilárd Kovács}

Jürgen Stabel

Mingmin Ren

AREVA, AREVA NP, Paul-Gossen-Strasse 100, 91052 Erlangen, Germany

Bruno Ladouceur

AREVA, AREVA NP, 10 Rue Juliette RECAMIER, 69456 Lyon, France

\section{Introduction}

In a nuclear reactor, heat is produced as a result of nuclear fission occurring inside the fuel rods. The heat from the fuel rods is transferred to water serving as reactor coolant to generate steam, either directly inside the reactor or in an external steam generator. The steam drives a steam turbine to generate electricity. To obtain a nuclear power plant with the highest possible power generating capacity, the reactor coolant must be capable of absorbing as much heat as possible. This can be achieved by means of a high coolant flow velocity and a high degree of coolant turbulence. Spacers fitted with vanes that impart swirl to the flow can be deployed to increase the turbulence. The coolant flow induces vibration of the fuel assemblies and their fuel rods at amplitudes in the micrometer range. Two different kinds of vibration arise: fuel assembly vibration and fuel rod vibration which may cause fretting wear [1] to the fuel rods at the locations at which they are supported.

Inside the reactor, neutron irradiation causes material transformation, in such a way at the fuel rod cladding tubes and the spacers. The types of irradiation-induced material transformation that can occur include transmutation and changes in the physical and mechanical properties of the materials [2]. Transmutation is due to the neutron-nucleus reaction.

The production of noble gases (e.g. He and $\mathrm{Ne}$ ) is the most significant factor governing transmutation. The atomic radius of a noble gas is larger than that of metals. As a result of this larger radius, the noble gas induces lattice defects in the metal, with the gas atoms either occupying a vacancy in the metal lattice or becoming deposited between the lattices (as interstitial atoms).

The above-mentioned changes in the physical and chemical properties of the materials can be caused by elastic collisions between neutrons and atomic nuclei, in which the neutrons displace the atomic nuclei from the lattice. The atomic nuclei themselves often have so much kinetic energy that they also displace other atomic nuclei from the lattice, leaving vacancies at these locations. The free atomic nuclei subsequently become deposited as interstitial atoms.

Both transmutation and the movement of atoms induce growth in the material [3]. This also leads to growth of the 
spacer grid. Inside the fuel rod, there is a small gap between the fuel pellets and the cladding tube allowing insertion of the pellets during manufacturing. Neutron irradiation does enhance on material creep (irradiation-induced creep) [3-5]. This leads to relaxation of the spacer springs and promotes permanent creep deformation of the cladding tube (reduction of diameter).

As a result of spacer growth, reduction in cladding tube diameter and spring relaxation, the initial preload (at BOL (beginning of life)) of the spacer springs decreases, sometimes leading to a gap between the fuel rod and the spacer spring [6].

The aim of the work presented here was to investigate the susceptibility of the fuel rods to fretting under a typical condition of support. The support configuration, which was analyzed under identical loads, featured different types of fit (interference fit due to spring preload and clearance fit with a gap). The duration of each fretting test was 100 hours.

\section{Description of Tests}

Before the actual fretting tests were conducted, the dynamic behavior of a test fuel assembly was investigated under representative conditions inside a flow channel with water [7]. In this channel it is possible to subject a full-size test assembly not only to flow in the main axial direction but also to transverse flows. The aim of this preliminary investigation was to record the fuel rod vibrations (vibration amplitude and frequency) so that they could then be used as a basis for generating the excitation signal for the subsequent fretting tests.

In the fretting tests described here, the same excitation signal spectrum was used each time in order to ensure comparability of the results. The fretting tests were performed inside an fretting test facility (autoclave) equipped with an electromagnetic excitation system. A fuel rod of shortened length was used as the test rod. The autoclave is designed for testing in air or in still water. The rod supports inside the autoclave are removable so that the fretting susceptibility can be investigated for different types of supports and fits (BOL and EOL (end of life)). The design of the autoclave allows the axial spacing (spans) between the supports (here: spacer cells) to be varied, thereby increasing the number of parameters to be investigated.

\subsection{Autoclave}

Fig. 1] shows the autoclave with its main components: test rod, spacer cells, EMAC (Electromagnetic Actuation Control) system, azimuthal stabilization system, Plexiglas box and displacement sensors. The test rod is supported in three spacer cells and is suspended from a thin wire that prevents axial movement of the rod. The Plexiglas box enables tests to also be carried out in still water. Furthermore, the displacement sensors, which measure the lateral oscillation of the test rod (in the direction of excitation as well as perpendicular to the direction of excitation), are mounted in the wall of this Plexiglas box.

The EMAC system, comprising electromagnets and a controller with signal generator, filter and amplifier, applies the

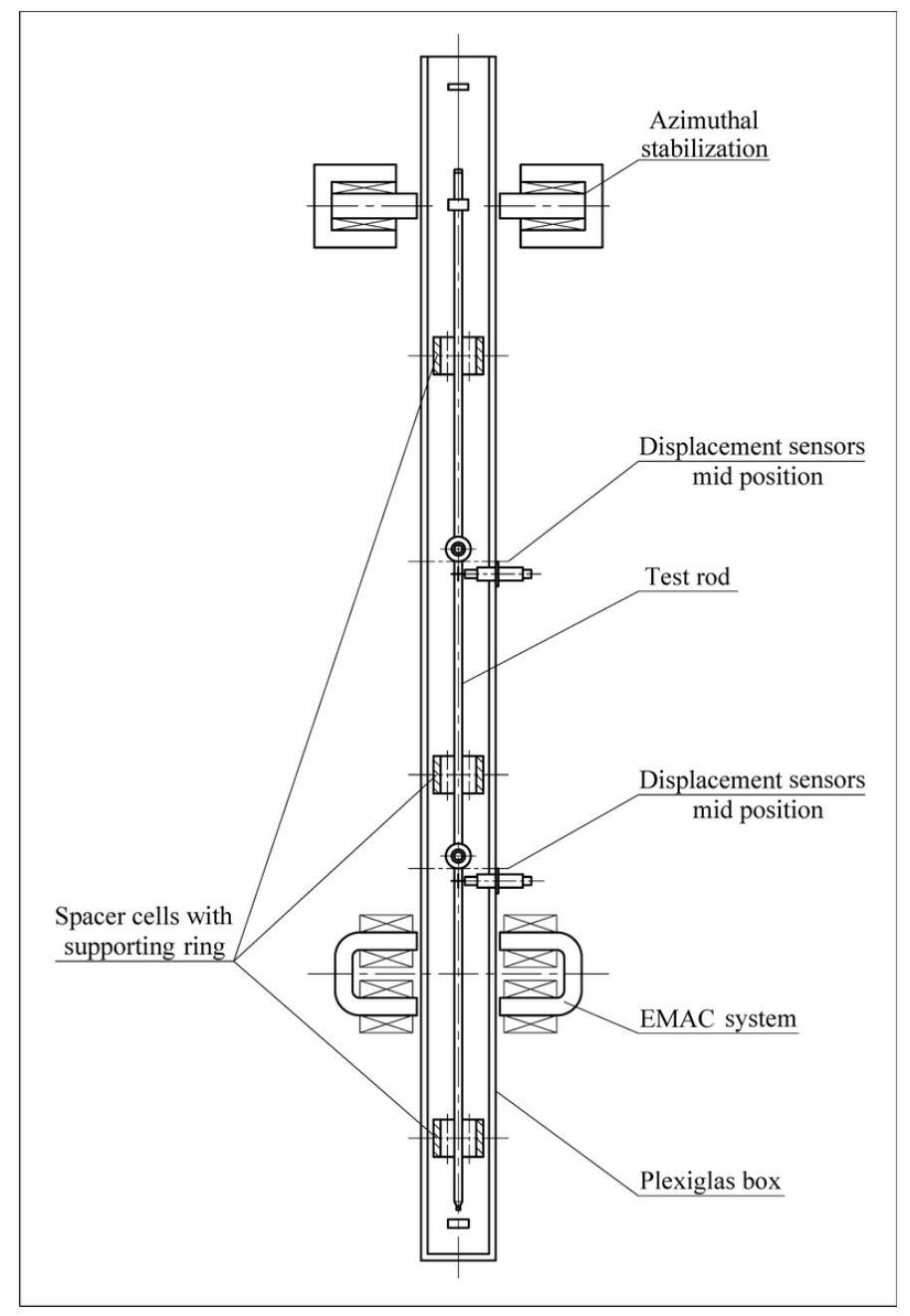

Fig. 1. Configuration of autoclave

electromagnetic input excitation to the test rod. The excitation signals that can be generated using the EMAC system can be varied as required. This high degree of flexibility permits simulation in the autoclave of the flow-induced vibrations encountered in the reactor core. The azimuthal stabilization system prevents uncontrolled rotation of the test rod about its longitudinal axis.

\subsection{Test Specimen}

Zirconium-alloy cladding tubes manufactured for actual fuel assemblies were used as the test rods for the fretting tests. Instead of uranium pellets, the cladding tubes were filled with molybdenum pellets to permit easy handling of the rods. Molybdenum pellets have a density similar to that of uranium pellets. To ensure reliability and reproducibility of the tests, each test rod was subjected to stringent criteria in terms of straightness, out-of-roundness and diameter.

The rod was supported at three locations by means of spacer mockups made from a zirconium alloy. The rod-to-grid fit was adjusted to simulate various service-life conditions (BOL and EOL). This was done by varying the so-called "clear" inside diameter of the support cells while keeping the rod diameter the same. Adjusting the fit between rod and support in this way also 
altered the spring preload. The fits used for the tests are shown in Tab. 1 Columns 4 and 5 of Tab. 1 indicate which tests were carried out with the respective types of fit.

\subsection{Test Procedure}

During the tests, the rod was supported at three axial elevations with the same type of fit. New support cells and new rods were used for each test. The same axial spans (distances between support elevations) were used in the series of the tests. All of the tests were carried out in water and at room temperature.

In addition to the fretting tests, vibration tests were also conducted to investigate the influence of the fit on the dynamic behavior of the rod. The objective of these vibration tests was to determine the resonant frequency and vibration amplitude. A turbulent signal was used as the excitation signal in both the vibration tests and the fretting tests. The current intensity of the EMAC system electromagnet coils is proportional to the induced excitation force. In all of the tests that were performed, the intensity of the excitation signal - in other words, the effective current intensity of the electromagnet coils - was the same. The standard spectrum of the excitation signal used in the tests has a frequency range of $5 \mathrm{~Hz}$ to $80 \mathrm{~Hz}$.

The vibration tests enabled the transfer function of the vibrating system to be determined on the basis of the measured lateral rod movements.

In contrast to the relatively short-duration vibration tests, each of the fretting tests lasted 100 hours. After each fretting test, the number, depth and volume of the fretting marks were measured. The depths and volumes of the fretting marks were measured using a computer-controlled interferometer. This optical instrument scans the entire rod surface and generates a threedimensional image of the worn surface. In addition, crosssectional profiles were produced of the fretting marks using a data analysis program.

\section{Results}

\subsection{Fretting Analysis}

Tab. 2 summarizes the results of the fretting tests. At BOL, the support springs exhibit a large preload. The initial spring preload, for example, was $44.5 \mathrm{~N}$. No identifiable fretting marks were discovered following the 100-hour fretting test. After the spring preload was reduced to $3.5 \mathrm{~N}$, the first fretting marks were then found after the respective 100-hour test. Most of these marks were faint surface imprints whose depth and volume were so small that they could not be measured (the volume of these fretting marks falls within the range of error of the interferometer and is therefore shown as $\sim 0 \mathrm{~mm}^{3}$ for purposes of simplification)

In addition to these imprints, a small number of measurable fretting marks having a maximum depth of $10 \mu \mathrm{m}$ were also identified. A further reduction in the spring preload to $0.6 \mathrm{~N}$ did not result in any significant differences in terms of the number and depth of the fretting marks in comparison to the previous measuring point.

Fig. 2 shows typical rod surface imprint of this kind. The line-type contact produces elongated marks having a very small width-to-length ratio (0.2-0.35).

The gradual development of a clearance fit between the spacer cell and the rod leads to an increase in the number of fretting marks. During this phase the volume of the marks also increases rapidly. The largest fretting mark depths that were measured -e.g. in the case of a clearance fit of $0.10 \mathrm{~mm}-$ amounted to $92 \mu \mathrm{m}$.

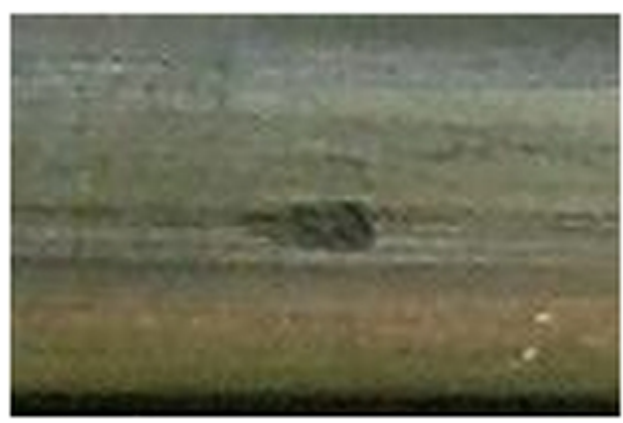

Fig. 2. Surface imprints

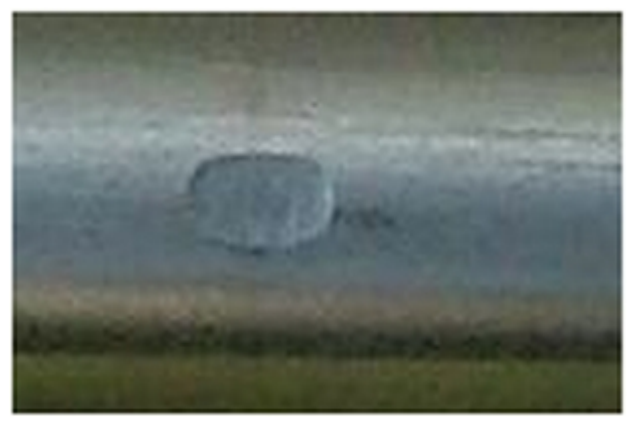

Fig. 3. Largest fretting marks

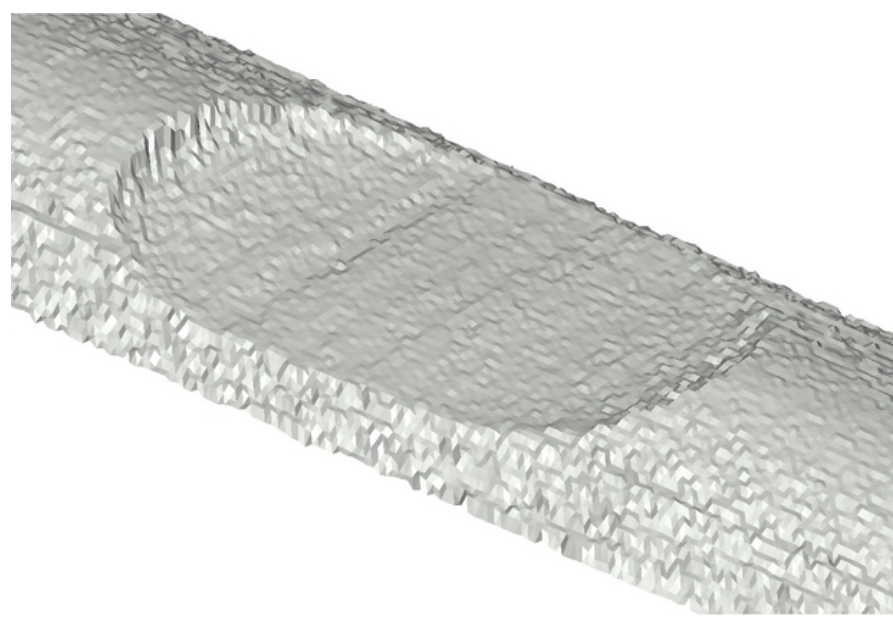

Fig. 4. 3-D images of deepest fretting mark

Fig. 3 shows a typical fretting mark in case of gapped support. Three-dimensional image of the deepest fretting mark, produced by means of surface measurements carried out using the interferometer (see Section 2.3), is shown in Fig. 4 The resulting 
Tab. 1. Vibration and fretting tests

\begin{tabular}{lrccc}
\hline Type of fit & Preload & Gap & Vibration tests & Fretting tests \\
\hline Interference fit & $44.5 \mathrm{~N}$ & - & + & + \\
Interference fit & $3.5 \mathrm{~N}$ & - & + & + \\
Interference fit & $2.3 \mathrm{~N}$ & - & - & + \\
Interference fit & $1.5 \mathrm{~N}$ & - & + & - \\
Interference fit & $1.0 \mathrm{~N}$ & - & + & - \\
Interference fit & $0.6 \mathrm{~N}$ & - & - & + \\
\hline Clearance fit & - & $0.01 \mathrm{~mm}$ & - & + \\
Clearance fit & - & $0.05 \mathrm{~mm}$ & - & + \\
Clearance fit & - & $0.07 \mathrm{~mm}$ & + & + \\
Clearance fit & - & $0.09 \mathrm{~mm}$ & - & + \\
Clearance fit & - & $0.10 \mathrm{~mm}$ & + & + \\
\hline
\end{tabular}

Tab. 2. Number of fretting marks and depth and volume of deepest fretting marks

\begin{tabular}{lcccccc}
\hline Type of fit & Preload & Gap & $\begin{array}{c}\text { Number } \\
\text { of marks }\end{array}$ & $\begin{array}{c}\text { Number of marks } \\
\text { with depth } \geq 10 \mu \mathrm{m}\end{array}$ & $\begin{array}{c}\text { Maximum depth of } \\
\text { deepest mark }[\mu \mathrm{m}]\end{array}$ & $\begin{array}{c}\text { Volume of deepest } \\
\text { mark }\left[\mathrm{mm}^{3}\right]\end{array}$ \\
\hline Interference fit & $44.5 \mathrm{~N}$ & - & 0 & 0 & 0 & $\approx 0$ \\
Interference fit & $3.5 \mathrm{~N}$ & - & 9 & 1 & 10 & 0.007 \\
Interference fit & $2.3 \mathrm{~N}$ & - & 6 & 3 & 13 & 0.020 \\
Interference fit & $0.6 \mathrm{~N}$ & - & 7 & 1 & 10 & 0.007 \\
\hline Clearance fit & - & $0.01 \mathrm{~mm}$ & 9 & 0 & $<10$ & 0.008 \\
Clearance fit & - & $0.05 \mathrm{~mm}$ & 9 & 0 & $<10$ & 0.010 \\
Clearance fit & - & $0.07 \mathrm{~mm}$ & 10 & 6 & 45 & 0.087 \\
Clearance fit & - & $0.09 \mathrm{~mm}$ & 11 & 6 & 85 & 0.187 \\
Clearance fit & - & $0.10 \mathrm{~mm}$ & 11 & 4 & 92 & 0.224 \\
Clearance fit & - & $0.10 \mathrm{~mm}$ & 12 & 9 & 55 & 0.171 \\
Clearance fit & - & $0.10 \mathrm{~mm}$ & 13 & 8 & 65 & 0.182 \\
\hline
\end{tabular}
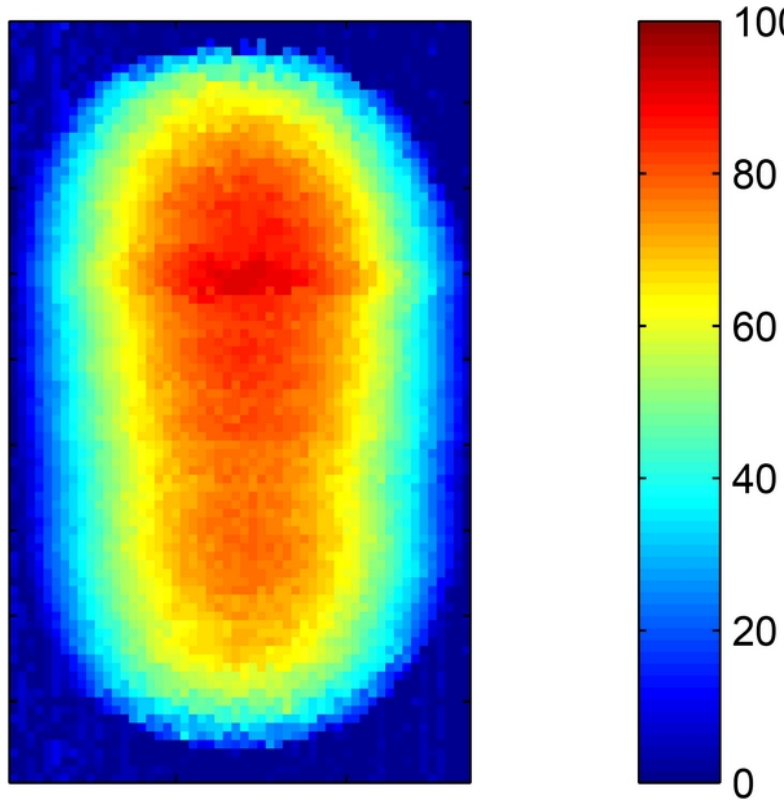

Fig. 5. Color-coded height image

top view is presented in the form of color-coded height image in Fig. 5 The fretting mark is an elliptical indentation caused by penetration of the truncated-cone-shaped dimple into the cylindrical rod, the axes of the rod and the truncated cone being in the same plane and approximately perpendicular to one another.

Fig. 6 shows the cross-sectional profile of the 3D image. The section plane is perpendicular to the cross-sectional area of the

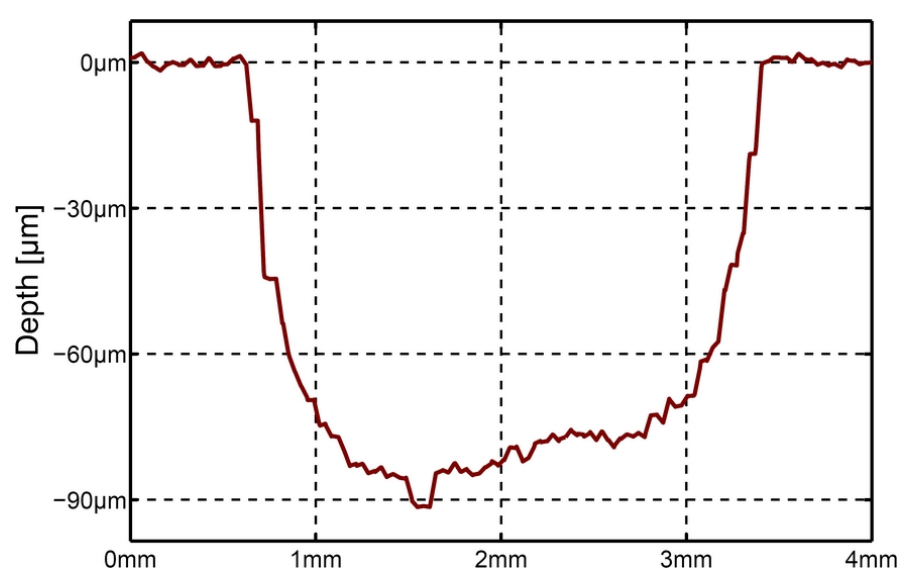

Fig. 6. Cross-sectional profile of deepest fretting mark

cylinder along the test rod axis. The width of the marks is mainly dependent on their depth.

The fretting test with a clearance fit of $0.10 \mathrm{~mm}$ was carried out a total of three times.

The fretting tests demonstrated that a large clearance between rod and support promotes fretting. As can be seen in Tab. 1. the largest grid-to-rod gap used in the tests was $0.10 \mathrm{~mm}$. The tests performed using this gap produced fretting mark volumes in the range of $0.171-0.224 \mathrm{~mm}^{3}$

Fig. 7 and Fig. 8 show the aggregate depth (sum of maximum depths of all marks) and the aggregate volume of the fretting marks as a function of the fit between rod and support. The fig- 
Aggregate fretting depth

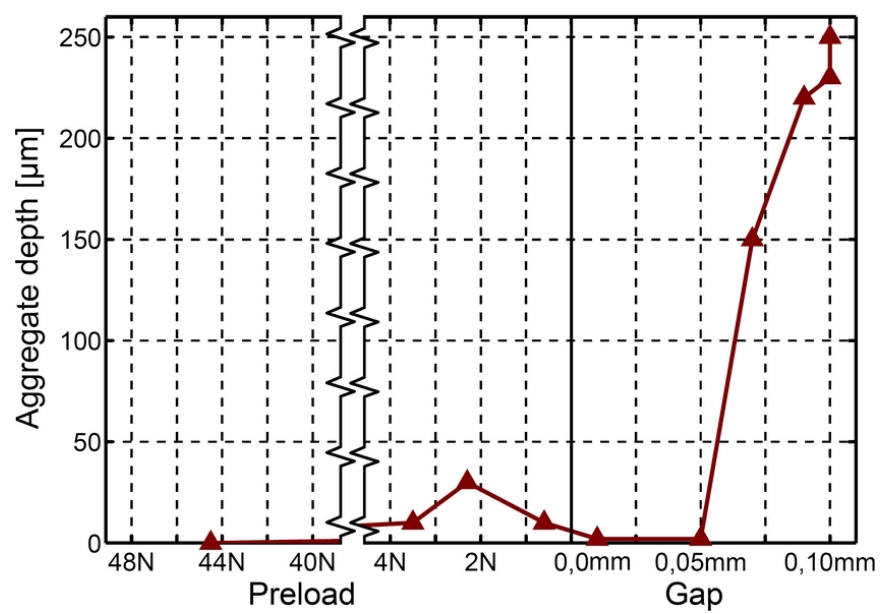

Fig. 7. Aggregate fretting depth

Aggregate fretting volume

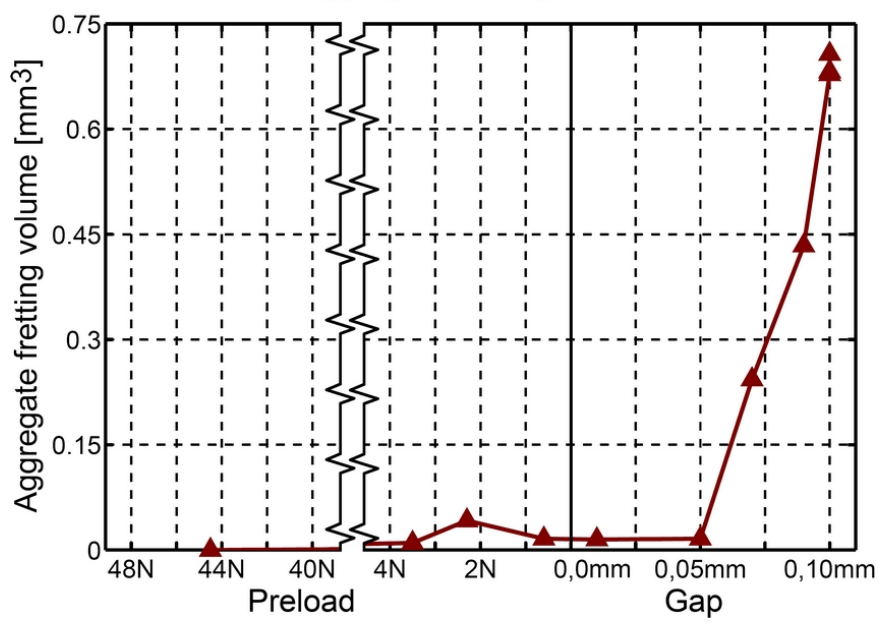

Fig. 8. Aggregate fretting volume

ures clearly show that accelerated fretting does not start until a sufficiently large gap has formed between the rod and the support (EOL condition). As this gap grows larger, fretting behavior changes and becomes progressive. In this case the susceptibility to fretting and hence the probability of fretting also increase, irrespective of the type of support configuration involved.

As has already been described, the fretting test with a clearance fit of $0.10 \mathrm{~mm}$ was repeated twice. The aggregate fretting volumes from all three tests are almost identical. This proves the high reproducibility and reliability of the tests performed.

\subsection{Vibration Analysis}

In order to obtain a better understanding of fretting behavior it is essential to carry out a dynamic analysis of the vibrating $\mathrm{rod} /$ support system since this rod vibration is in relation with fretting. During the vibration tests, the lateral vibrations of the rod in the direction of excitation and perpendicular to the direction of excitation were recorded. These two vibration signals were each measured at two different elevations on the rod longitudinal axis (see Fig. 1), one of these planes being situated in the bottom span (between the bottom and middle supports) and one in the top span (between the middle and top supports).
RMS vibration amplitudes

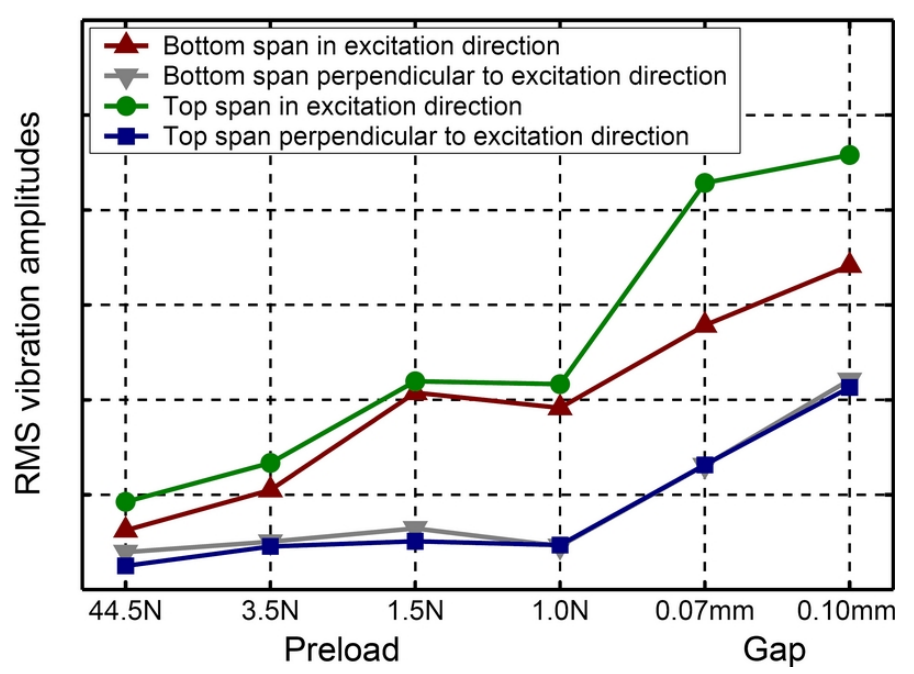

Fig. 9. Rms vibration amplitude

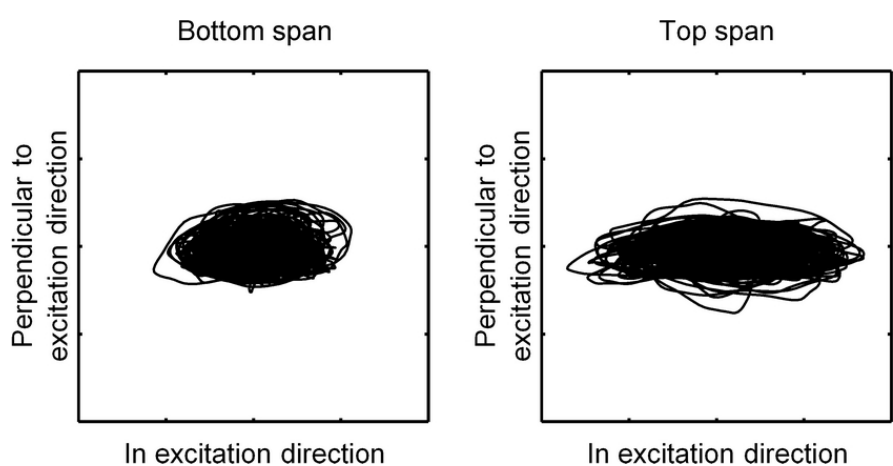

Fig. 10. Examples of root locus curves

The excitation signal was a turbulent signal with a standard spectrum of $5 \mathrm{~Hz}-80 \mathrm{~Hz}$. Consequently, the system response also comprises turbulent signals (lateral vibration signals). This means that the displacement signal can be described by stating its effective value, the root-mean-square (rms) vibration amplitude.

Fig. 9 presents the rms vibration amplitudes for all four displacement signals as a function of fit. The figure shows that the rms amplitude, i.e. the intensity of the vibration, increases as the gap becomes larger. If the spacer springs have a preload, the vibrations in the direction of excitation are dominant; i.e. the rms amplitudes of the transverse vibrations are much smaller than those of the vibrations in the direction of excitation. In this case, only sliding and tilting motion can occur.

As soon as a clearance fit has formed, the rod is able to move more freely. It can move horizontally within the spacer cell, it can rotate about its own axis, and perpendicularly about it too. At a spring or dimple, impact as well as sliding and tilting motion can occur. Hence the rod is capable of moving in a much more complex and chaotic fashion. This explains why not only the vibration motions in the direction of excitation but also those in the transverse direction increase substantially as the clearance fit becomes larger. It can be observed that the difference between the rms vibration amplitude in the direction of excitation and 
that in the transverse direction is larger in the top span than in the bottom span. This is due to the fact that the excitation system is located in the bottom span.

Fig. 10 illustrates this effect (condition) by giving examples of root locus curves for the top and bottom spans.

Beside the vibration amplitude of the rod - to describe its dynamic behavior - the transfer function of the vibrating system can also be used. In the system under consideration here, the excitation current intensity, which is proportional to the excitation force, is the input signal, and the rod motions are the output signals.

Fig. 11 presents the transfer functions for rod motion in the direction of excitation. Since the excitation system was located in the top span for the tests, only the motions of the bottom span were examined in this case. Analysis of the transfer function in the frequency range of $5 \mathrm{~Hz}-80 \mathrm{~Hz}$ shows that the resonant frequency drops as the gap increases. Initially (at BOL), with a preload force of $44.5 \mathrm{~N}$, the resonant frequency is around $60 \mathrm{~Hz}$. With the maximum clearance of $0.10 \mathrm{~mm}$ (EOL condition), the resonant frequency is $28 \mathrm{~Hz}$. As core residence time increases (during which time the preload decreases and the gap grows larger), a second resonant frequency, which lies above the first, appears starting from a certain point.

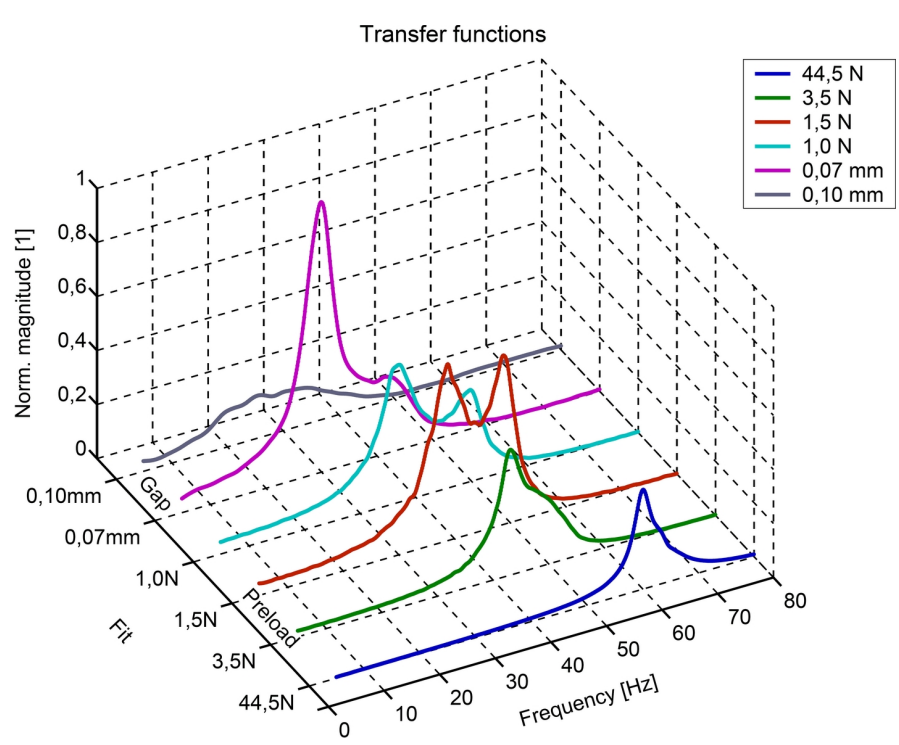

Fig. 11. Transfer functions; bottom span, in direction of excitation

Fig. 11 shows that this point lies at spring preloads of between $3.5 \mathrm{~N}$ and $1.5 \mathrm{~N}$. After reaching a peak at $1.5 \mathrm{~N}$, the magnitude of this resonant frequency decreases in the course of further core residence to a value that is difficult to distinguish from the ambient spectrum. Like the first resonant frequency, the second resonant frequency also decreases as the preload decreases and the gap becomes larger, but to a lesser degree.

The appearance of the second resonant frequency and the decrease in the resonant frequencies can be considered to be due to the reduction in stiffness of the rod/support system and the strongly non-linear characteristics of the vibrating system. Fig. 11 confirms this, and shows that the magnitudes of both resonant frequencies decrease significantly as a result of only a slight change in spring preload (from $1.5 \mathrm{~N}$ to $1.0 \mathrm{~N}$ ). As the stiffness of the vibrating system decreases, the rod is able to move more freely and more extensively at the same level of excitation. Larger vibrations are more strongly damped by the surrounding water.

Due to the non-linear vibration behavior, the shape of the transfer function also alters as the fit changes. In contrast to the frequency curves with sharp peaks obtained when a spring preload is present (see Fig. 11. F = 44.5 N), the curves obtained when there is a large gap have a squat, flattened shape. Under these circumstances, the resonant frequencies can only be determined with a considerable degree of uncertainty.

For the purpose of further analysis of the vibrating system, the phase curves of all transfer functions were calculated. The phase diagrams (not shown in this paper) illustrate how the rod moves in the top span relative to the bottom span. At the first resonant frequency, the rod vibration in the top span is in phase opposition with that in the bottom span. In the case of the second resonant frequency, by contrast, the vibrations of the top and bottom span are in-phase. The phase diagram when a gap is present also shows the existence of a rigid rod motion component (phase difference $=0$ ). However, its frequency cannot be clearly detected in the transfer function because the modal resonance curves are superimposed on one another.

These results are confirmed by the mode shapes resulting from the numerical calculation. Fig. 12 presents the first and second mode shapes determined by numerical analysis.

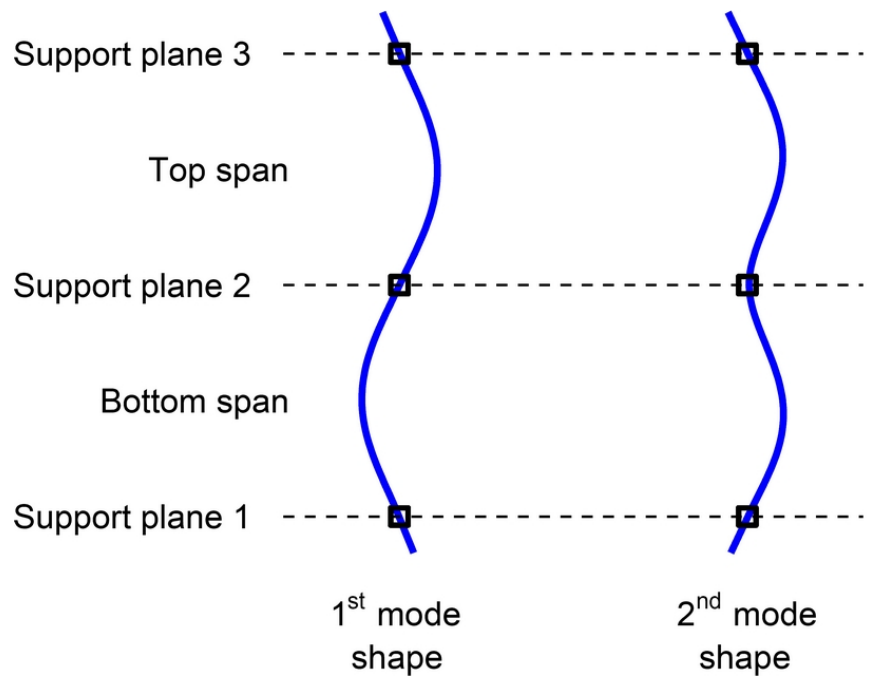

Fig. 12. Mode shapes

\section{Conclusions}

The changes occurring at the fit between the fuel rod and the spacer grid during the fuel residence time in the core promote fretting. Under reactor operating conditions the continuously diminishing interference fit can develop into a clearance fit. The influence of fit on fretting behavior was investigated by performing model tests on a typical fuel rod support configuration. A 
zirconium-alloy test rod was used for the tests. The rod was suspended from a wire inside a water-filled autoclave and supported at three axial elevations. A support type was subjected to 100-hour fretting tests to investigate the influence of different fits. The tests were carried out at room temperature. After the tests, the depth and volume of the fretting marks were measured. In order to investigate the influence of fit on the dynamic behavior of the vibrating rod/support system, vibration measurements were also taken in the autoclave in addition to the fretting tests.

From these vibration measurements it was found that the stiffness of the vibrating system decreases as spacer spring preload decreases and the gap between the rod and the spacer spring increases. With a clearance fit, the test rod is able to move more freely and more extensively at the same level of excitation. As a result of the reduction in stiffness, the resonant frequencies are also reduced. The rods, exhibiting more pronounced vibration in the clearance fit, are more strongly damped. In the case of a clearance fit of $0.10 \mathrm{~mm}$, the first resonant frequency is dominant, being in the range of $25 \mathrm{~Hz}-30 \mathrm{~Hz}$. As the gap becomes larger, the magnitude of the higher resonant frequencies decreases until they are difficult to distinguish from the spectrum. With a clearance fit, the intensity of rod motion increases not only in the direction of excitation but also perpendicular to the direction of excitation. Rod motion is considerably more complex and chaotic under clearance fit conditions than with an interference fit, this being particularly evident from the impacts occurring between rod and support.

The test results confirmed that the fretting wear increases with the growth of rod energy of motion. The fretting test conducted on a test rod subjected to a spring preload of $44.5 \mathrm{~N}$ did not produce any identifiable fretting marks after testing for 100 hours. The first small marks, with a maximum depth of $13 \mu \mathrm{m}$, did not start to appear until spring preload had been reduced to approximately $0.5-3.5 \mathrm{~N}$. The number, depth and volume of the fretting marks increased as the grid-to-rod gap became larger. In summary, it can be stated that the extent of fretting and the susceptibility to fretting increase as spring preload decreases and the gap increases.

It must be remembered that these were model tests in which all parameters, with the exception of fit, remained constant. Using other contact geometries could lead to shifting the fretting wear limit on either side but would likely show the same tendency. The results obtained can play an important role in the future development of spacer grids with respect to prevent fretting. The methodology described here can be used to assess the fretting susceptibility of new spacer grid designs. Although the test conditions are not identical to conditions inside the reactor, the test results can help provide a better understanding of real fretting processes. Research in the field of fuel assembly spacer design is of vital importance since these findings can help extend the service lives of fuel assemblies in the core.

\section{References}

1 Waterhouse RB, Fretting Corrosion, Pergamon Press, Oxford, 1975.

2 Csom Gy, Atomerômúvek üzemtana, I. kötet, A reaktorfizika és -technika alapjai, Múegyetemi Kiadó; Budapest, 1997.

3 Frost B.R.T, A Comprehensive Treatment, Materials Science and Technology, Nuclear Materials, Volume 10B(Part II, VCH Verlagsgesellschaft mbH,Weinheim), (1994), 29-33.

4 Keller C, Möllinger H, Kernbrennstoffkreislauf, Band 1, Allgemeine Aspekte. Versorgung von Kernkraftwerken, Dr Alfred Hüting Verlag, Hiedelberg, (1978), 207-208.

5 Epprecht W, Werkstoffkunde der Kerntechnik, Birkhäuser Verlag, Basel, (1961), 240-242.

6 Billerey A, Evolution of fuel rod support under irradiation - Impact on the mechanical behaviour of fuel assemblies, in: Structural behaviour of fuel assemblies for water cooled reactors, in Cadarache, France 22-26 November 2004, (2005), 101-111. IAEA-TECDOC-1454, Vienna.

7 Stabel J, Koebke K, Stephens LG, Experimental and Analytical Procedures for Approving Fretting-Free Fuel Rod Support, (1999). LWR Conference Utah. 